\title{
Preparation of L-cysteine Salicylaldehyde Schiff-base Modified Macroporous Polystyrene Resin and Its Application to Determination of Trace Cadmium and Lead in Environmental Water Samples
}

\author{
Fazhi Xie****, Fengjun Zhang*, Han Xuan*, Yejun Ge*, Yin Wang*, Guolian Li*, \\ Lei Zhu***;, and Won-Chun Oh***,it \\ *School of Materials Science and Chemical Engineering, Anhui Jianzhu University, Hefei 230022, China \\ **School of Earth and Space Sciences, University of Science and Technology of China, Hefei 230026, China \\ ***Department of Advanced Materials Science \& Engineering, Hanseo University, Seosan 356-706, Korea
}

(Received August 29, 2013; Revised January 16, 2014; Accepted July 18, 2014)

\begin{abstract}
In this work, a new method that utilizes L-cysteine salicylaldehyde Schiff-base modified macroporous polystyrene resin (PSCSC) as an effective sorbent has been developed for preconcentration of trace cadmium and lead in environmental water samples. The effect of $\mathrm{pH}$, the contact time, the elution conditions, the flow rate, the initial concentration of target metal ions, and the effects of interfering ions on the preconcentration of the analytes were investigated. The maximum adsorption capacity of PSCSC under optimum conditions for cadmium and lead were found to be $6.03-18.17 \mathrm{mg} / \mathrm{g}$ and $12.58-36.13 \mathrm{mg} / \mathrm{g}$ when the initial concentration of metal ions between $5.0-90 \mathrm{mg} / \mathrm{L}$. The limits of detection for cadmium and lead were $2.46 \mathrm{ng} / \mathrm{L}$ and $0.52 \mu \mathrm{g} / \mathrm{L}$, with a preconcentration factor of 200 . The developed method has been validated by analyzing certified reference material and successfully applied for the enrichment and determination of trace cadmium and lead from environmental water samples.
\end{abstract}

Key words : L-cysteine salicylaldehyde Schiff-base, Macroporous polystyrene resin, Cadmium, Lead, Preconcentration

\section{Introduction}

C admium and lead have been well recognized for their negative effects on the environment. Both of these heavy metal ions can accumulate readily in living systems, and this accumulation can have adverse effects on metabolic processes. ${ }^{1-2)}$ Flame atomic adsorption spectrometric (FAAS) analysis is one of the most popular techniques for determination of the presence of these heavy metal ions because of its high specificity and low cost. However, process sensitivity is usually insufficient for the determination of trace metal ions in environmental samples. ${ }^{3)}$ Due to the complexity of the sample matrix and low concentrations of cadmium and lead found in environmental water samples, ${ }^{3-5}$ an efficient preconcentration step before level determination is required. ${ }^{6}$ Several methods, such as co-precipitation, ${ }^{7,8)}$ liquid-liquid extraction, ${ }^{9,10)}$ cloud point extraction ${ }^{11,12)}$ and solid phase extraction ${ }^{6,13)}$ have been used for the enrichment of trace cadmium and lead from environmental samples before sample analysis using flame atomic absorption

\footnotetext{
${ }^{\dagger}$ Corresponding author : Fazhi Xie

E-mail : fzxie@mail.ustc.edu.cn

Tel : +86-551-6382-8262 Fax : +86-551-6382-8106

${ }^{\dagger}$ Corresponding author: Won-Chun Oh

E-mail : wc_oh@hanseo.ac.kr

Tel : +82-41-660-1337 Fax : +82-41-688-3352
}

spectrometry techniques. For its exhibited simplicity, its flexibility in allowing an analysis of the solid phase, its high preconcentration factor, and its low cost and extraction time for sample preparation, the solid phase extraction approach has gained rapid acceptance..$^{3)}$ Macroporous polystyrene resin is a frequently used resin in such solid phase preconcentration procedures, due to its good physical and chemical properties such as porosity area, high surface area, durability, and easy modification. ${ }^{14)}$ In the present paper, a new chelating solid phase extractant on the base of a macroporous polystyrene resin chemically modified with L-cysteine salicylaldehyde Schiff-base (PS-CSC) was synthesized and a simple solid phase extraction procedure was developed for simultaneous preconcentration of trace cadmium and lead in environmental samples.

\section{Experimental Procedure}

\subsection{Apparatus}

A flame atomic absorption spectrometer from the Beijing Rayleigh Analytical Instrumentation Corporation (model WFX-1E2, Beijing, China), equipped with an air-acetylene flame and lead and cadmium hollow cathode lamps was used for metal ion analysis. The $\mathrm{pH}$ measurements were conducted using a PHS-2C pH-meter (Dazhong Instrumentation Corp., Limited. Shanghai, China). Infrared measurements were performed using a model WQF-300 spectrometer (Beijing, China) and KBr pellets. 


\subsection{Reagents}

The macroporous polystyrene resin was X-5 resin (Chemical Industrial Company affiliated with Nankai University, Tianjin, China). L-cysteine and salicylaldehyde was purchased from Shanghai Chemical Reagent Corporation, China. The stock solutions of metal ions $(1.0 \mathrm{~g} / \mathrm{L})$ were prepared from analytical corresponding reagent grade metal salts in nitric acid, hydrochloric acid, or sulfuric acid by dissolving the appropriate amounts in distilled water. All working solutions were diluted according to experimental requirements. Dilute $\mathrm{HNO}_{3}$ and $\mathrm{NaOH}$ were used for $\mathrm{pH}$ adjustment. All chemicals used were of analytical reagent grade.

\subsection{Preparation of PS-CSC}

L-cysteine salicylaldehyde Schiff-base was synthesized according to the process detailed in the literature. ${ }^{15)}$ Macroporous polystyrene resin was nitrated, aminated, diazotized and then coupled with L-cysteine salicylaldehyde Schiffbase at $0-5^{\circ} \mathrm{C}$ to prepare the adsorbent. ${ }^{16)}$ The resulting reddish brown beads were filtered, water washed to neutral $\mathrm{pH}$, and finally vacuum dried for further use.

\subsection{Batch experiments}

PS-CSC (0.1 g) was equilibrated with a suitable amount of metal ion solution at a certain $\mathrm{pH}$ in a glass stoppered bottle $(250 \mathrm{~mL})$ for a fixed period of time; the unextracted metal ion in the solution phase was measured using FAAS. This method was used to investigate the effect of shaking time and the maximum adsorption capacities of cadmium and lead on PS-CSC.

\subsection{Column experiments}

PS-CSC (2.0 g) was packed in a glass tube $(200 \mathrm{~mm} \times$ 10 mmi.d.) and a solution containing metal ions was adjusted to a suitable $\mathrm{pH}$ and percolated at a certain flow rate. The metal ions retained on the column were eluted with a suitable eluent and that determined using FAAS.

\subsection{Samples and sample preparation}

$0.500 \mathrm{~g}$ of aquatic sediment reference material (GBW 07350) was added to a closed polytetrafluoroethylene beaker. Then, 7, 4, and $4 \mathrm{~mL}$ of concentrated $\mathrm{HNO}_{3}, \mathrm{HClO}_{4}$, and $\mathrm{HF}$ were added, respectively. The mixture was heated at $368 \mathrm{~K}$ for $12 \mathrm{~h}$ and evaporated nearly to dryness. After cooling, the residue was dissolved to $50 \mathrm{~mL}$ of $1.0 \mathrm{~mol} \cdot \mathrm{L}^{-1} \mathrm{HCl}$, and the solution was filtered. The environmental water samples were collected from Shiwuli River, Yi Lake, and Dongpu Reservoir (Hefei, Anhui, China), and then acidified with $2 \% \mathrm{HNO}_{3}$ and filtered with a $0.45 \mu \mathrm{m}$ filter; the filtrate was stored in glass bottles. The samples were analyzed within 3 days of collection.

\section{Results and Discussion}

\subsection{FTIR analysis of PS-CSC}

The proposed structure of PS-CSC is shown in Fig. 1; it

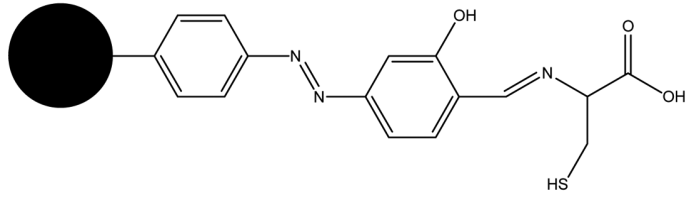

Fig. 1. Proposed structure of PS-CSC.

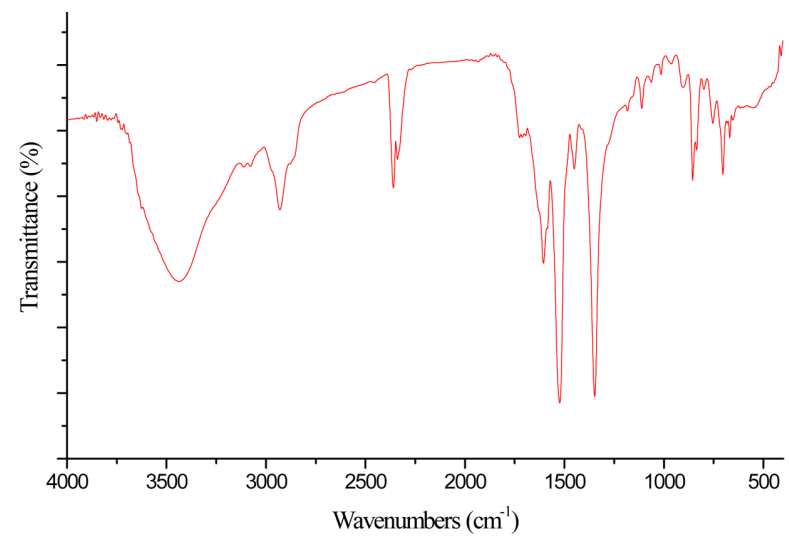

Fig. 2. FTIR of PS-CSC.

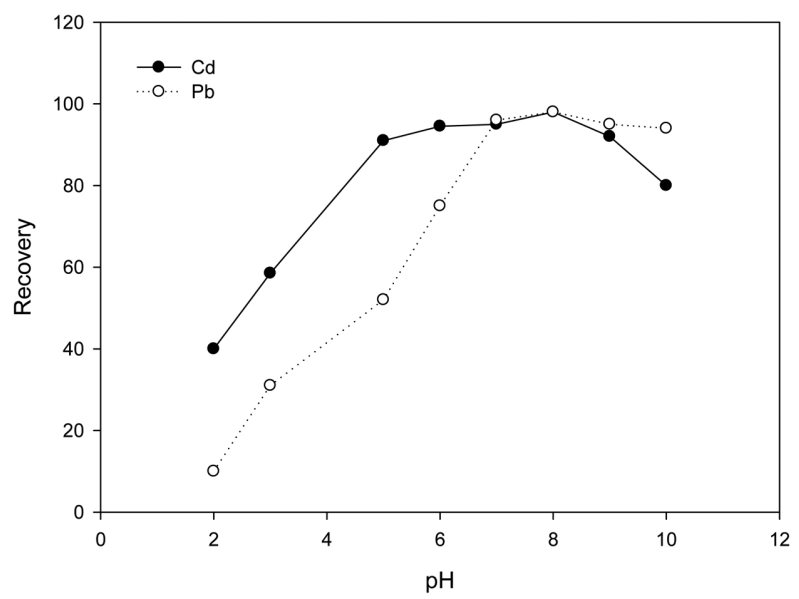

Fig. 3. Effect of $\mathrm{pH}$.

was characterized by FTIR analysis. Fig. 2 shows the FTIR spectrum of PS-CSC. The broad spectral band at $3400 \mathrm{~cm}^{-1}$ was found to correspond to $\mathrm{OH}$ - and $\mathrm{NH}$ - vibrations. The strong band at $2926 \mathrm{~cm}^{-1}$ is $\mathrm{CH}$-vibration. The bands that appeared at 1609 and $1449 \mathrm{~cm}^{-1}$ were contributed by $\mathrm{C}=\mathrm{N}$ and $-\mathrm{N}=\mathrm{N}$ - stretching, respectively. These results show that L-cysteine salicylaldehyde Schiff-base had been grafted onto the surface of macroporous polystyrene resin after modification.

\subsection{Effect of $\mathrm{pH}$}

The adsorption levels of cadmium and lead on PS-CSC in aqueous solutions with different $\mathrm{pH}$ values were examined by column experiment. Sorption and desorption flow rate was fixed at $2.0 \mathrm{~mL} / \mathrm{min}$. The extracted metal ions were eluted using $15 \mathrm{~mL}$ of $\mathrm{HCl}(6.0 \mathrm{~mol} / \mathrm{L})$ and determination by 


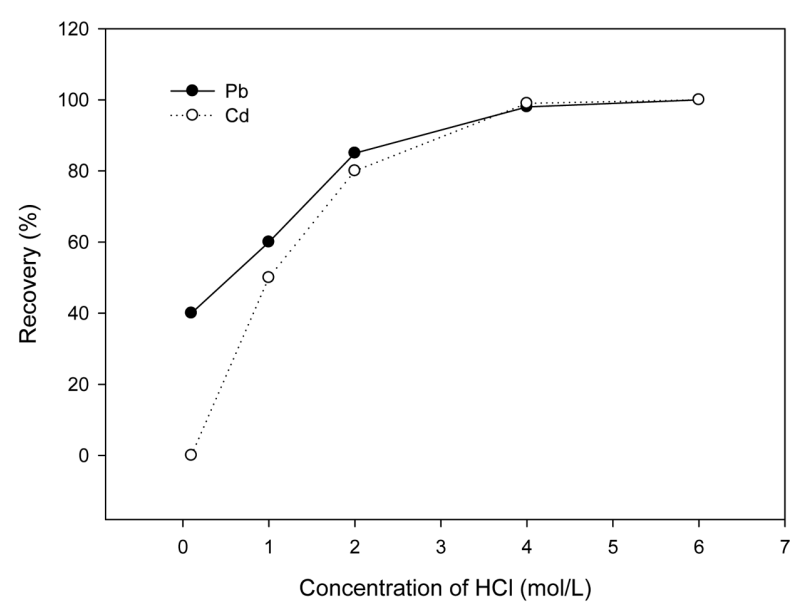

Fig. 4. Effect of concentration of $\mathrm{HCl}$ on the recovery of cadmium and lead.

FAAS gave the percentages of recovery of the eluted metal ions at various $\mathrm{pH}$ values (Fig. 3). Retention experiments were carried out in triplicate to determine the precision of the method. It was found that the retention behavior for both metal ions varies significantly with the changes of $\mathrm{pH}$. The optimum adsorption $\mathrm{pH}$ ranges are $5.0-8.0$ and 7.0 10.0 for cadmium and lead (Recovery $>95 \%$ ), respectively. The adsorbed metal ions cannot be desorbed by distilled water, and therefore the chelating process can be seen to contribute predominantly to the retention of these heavy metal ions. Therefore, $\mathrm{pH} 7.0$ was chosen for the preconcentration of cadmium and lead in the following experiments.

\subsection{Effect of eluent}

The effects of different organic and inorganic acids, such as $\mathrm{HCl}, \mathrm{HNO}_{3}$, EDTA, and citric acid, on the desorption of cadmium and lead from the column were determined at different concentrations using $15 \mathrm{~mL}$ volumes of various eluents. The results show that the use of an organic acid such as EDTA or citric acid does not give quantitative results (Recovery $>95 \%$ ). It was possible to quantitatively desorb the analyte with $\mathrm{HCl}$ and $\mathrm{HNO}_{3}$. The different concentrations of $\mathrm{HCl}$ for the recovery of both metal ions (Fig. 4) revealed that at least $4 \mathrm{~mol} / \mathrm{L} \mathrm{HCl}$ can lead to quantitative desorption. The more concentrated $\mathrm{HCl}$ solutions did not change the elution yield or elution kinetics.

\subsection{Effect of flow rate}

Figure 5 shows the effect of flow rate on the retention of cadmium and lead on PS-CSC. The optimum flow rates (Recovery $>95 \%$ ) for cadmium and lead extraction were less than 2.0 and $4.0 \mathrm{~mL} / \mathrm{min}$, respectively. To avoid a tedious extraction process, the flow rate of less than $1.0 \mathrm{~mL} / \mathrm{min}$ was not employed. For the desorption process, both metal ions can be desorbed quantitatively at a flow rate of $1.0-2.0 \mathrm{~mL} /$ $\min$. Above $2.0 \mathrm{~mL} / \mathrm{min}$, the recovery levels for both heavy metal ions were less than $90 \%$.The flow rate was maintained at $2.0 \mathrm{~mL} / \mathrm{min}$ throughout the column experiment.

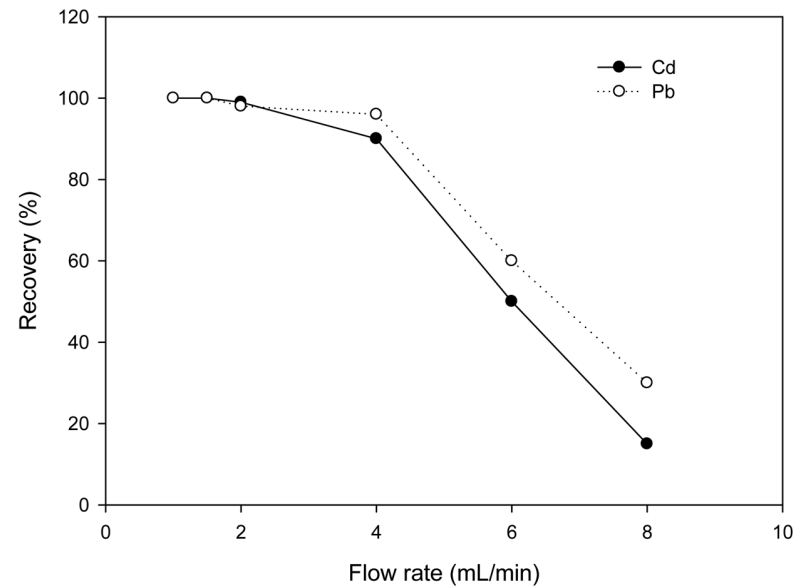

Fig. 5. Effect of retention flow rate on the recovery of cadmium and lead.

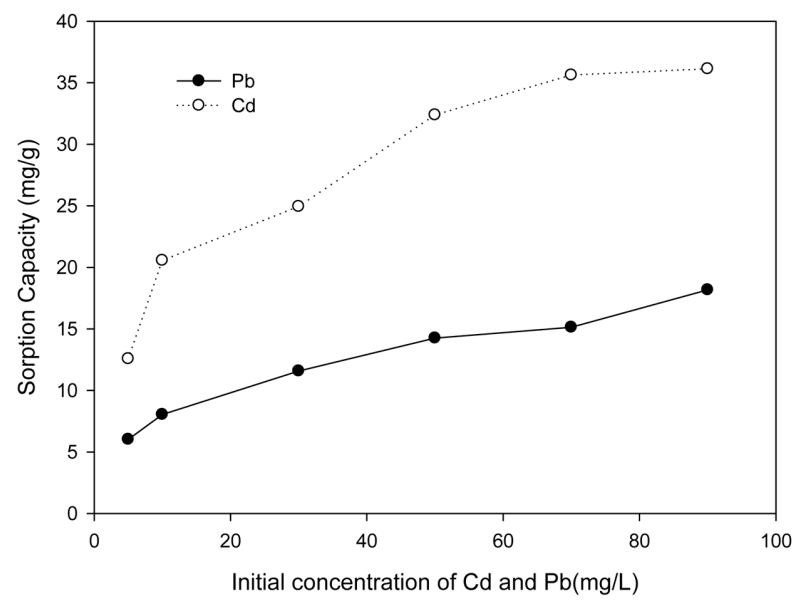

Fig. 6. Effect of initial concentration of cadmium and lead.

\subsection{Effect of initial concentration}

The adsorption levels of cadmium and lead on PS-CSC in aqueous solutions with different initial concentrations were examined using a bath experiment. It was found that the adsorption of cadmium and lead onto PS-CSC was strongly dependent on initial metal ion concentration (Fig. 6). The adsorption capacity of cadmium and lead on PS-CSC was found to increase dramatically with increasing of the initial concentration $(5-50 \mathrm{mg} / \mathrm{L})$, and then to increase slowly at high concentrations ( $50-90 \mathrm{mg} / \mathrm{L}$ ). This can be explained by the saturation of the surface sites of PS-CSC. The sorption capacity values for cadmium and lead were found to be 6.03$18.17 \mathrm{mg} / \mathrm{g}$ and $12.58-36.13 \mathrm{mg} / \mathrm{g}$ when the initial concentration was between $5.0-90 \mathrm{mg} / \mathrm{L}$ under the optimum conditions. This is satisfactory for the trace analysis of both heavy metals in aqueous solutions.

\subsection{Sorption kinetics}

PS-CSC (0.1 g) was equilibrated by shaking with $200 \mathrm{~mL}$ of a solution containing $50 \mathrm{mg} / \mathrm{L}$ of cadmium and lead for different time intervals $(2,7,10,20,30,40,50,60$, and $100 \mathrm{~min}$ ) and the recommended batch experiment was 
applied. The profile of sorption capacity for both heavy metal ions as a function of time is shown in Fig. 7. It is notable that the recovery levels of cadmium and lead can reach $80 \%$ in $15 \mathrm{~min}$. The fast extraction rate indicates that PS$\mathrm{CSC}$ is a reliable adsorbent for the preconcentration of trace cadmium and lead from aqueous solutions.

\subsection{Interference studies}

The effects of coexisting ions, such as $\mathrm{K}(\mathrm{I}), \mathrm{Na}(\mathrm{I}), \mathrm{Ca}(\mathrm{II})$, and $\mathrm{Mg}$ (II) on the preconcentration of cadmium and lead were studied using a column experiment. The tolerance limits of the coexisting ions were defined as the maximum concentration of the foreign substances that could cause an approximately $\pm 5 \%$ relative error in the determination. The tolerance limits for the heavy metal ions were found to be $2.0 \mathrm{~mol} / \mathrm{L}$ for $\mathrm{Na}(\mathrm{I})$ and $\mathrm{K}(\mathrm{I})$, and $0.8 \mathrm{~mol} / \mathrm{L}$ for $\mathrm{Ca}(\mathrm{II})$ and $\mathrm{Mg}$ (II). These results show that the presence of a solid phase matrix might be used to enrich cadmium and lead from environmental water samples, as the common constituents at normal levels do not adversely affect the adsorption efficiency of the matrix. The reusability of the present

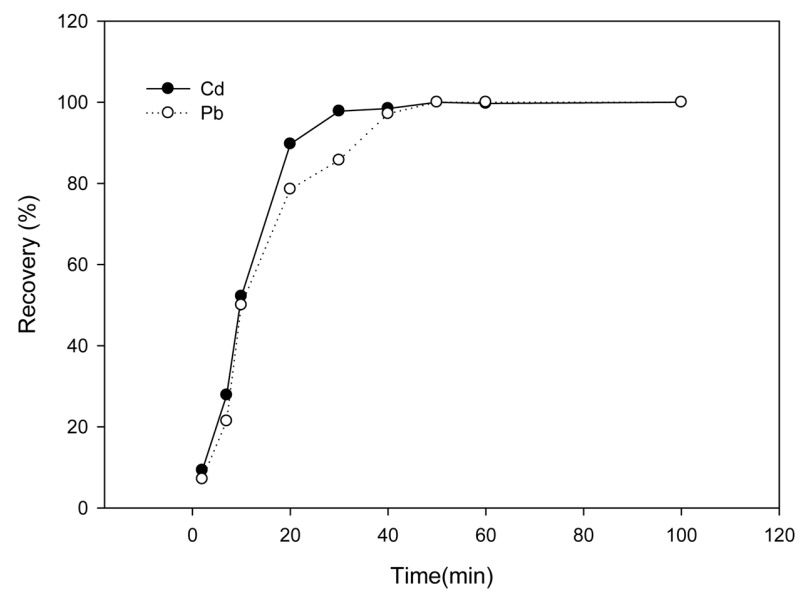

Fig. 7. Effect of contact time. matrix was examined after several loading and elution cycles. Trace amounts of cadmium and lead in environmental water samples can be quantitatively retained after 20 repeated uses and the sorption recovery can reach $96.80 \%$.

\subsection{Analytical performance}

The analytical performance of the method was examined by column experiment. The limits of detection (LODs) of cadmium and lead were below $2.46 \mathrm{ng} / \mathrm{L}$ and $0.52 \mu \mathrm{g} / \mathrm{L}$, respectively (calculated as 3 times the standard deviation of 11 replicate runs of procedure blanks). The relative standard deviation of the methods for cadmium and lead were $3.55 \%$ and $5.27 \%$, demonstrating good method precision. The maximum preconcentration factor for both heavy metal ions was 200.

To confirm the reliability of the method for the analysis of trace cadmium and lead in environmental samples, an aquatic Sediment reference material (GBW 07350, China) was used for method validation. Under optimum experimental conditions, the determined concentrations of cadmium and lead were $0.40 \pm 0.10 \mu \mathrm{g} / \mathrm{g}$ and $28.2 \pm 3.5 \mu \mathrm{g} / \mathrm{g}$, respectively (certified value: $\mathrm{Cd} 0.46 \pm 0.05 \mu \mathrm{g} / \mathrm{g} ; \mathrm{Pb} 28.5 \pm$ $2.9 \mathrm{\mu g} / \mathrm{g})$. Good agreement was obtained between the content estimated using the proposed method and the certified values for both metal ions. The results also indicate that the developed enrichment method for cadmium and lead are not affected by potential interference factors in real environmental samples.

\subsection{Analysis of environmental water samples}

Under optimum conditions, natural water samples (1000 $\mathrm{mL}$ ) were passed through the column for preconcentration and direct determination of both the target metal ions. Satisfactory results are shown in Table 1 . The method developed in the present paper for preconcentration and determination of trace amounts of cadmium and lead in environmental water samples has been proved to be reliable.

Table 1. Determination of Cadmium and Lead in Environmental Water Samples

\begin{tabular}{|c|c|c|c|c|}
\hline Samples & Metal ions & Added $(\mu \mathrm{g} / \mathrm{L})$ & Found $(\mu \mathrm{g} / \mathrm{L})$ & Recovery (\%) \\
\hline \multirow{4}{*}{ Shiwuli River } & \multirow{2}{*}{$\mathrm{Cd}$} & 0.00 & 3.76 & \\
\hline & & 5.00 & 8.60 & 96.80 \\
\hline & \multirow{2}{*}{$\mathrm{Pb}$} & 0.00 & 10.08 & - \\
\hline & & 5.00 & 14.82 & 94.80 \\
\hline \multirow{4}{*}{ Dongpu Reservoir } & \multirow{2}{*}{$\mathrm{Cd}$} & 0.00 & 2.19 & - \\
\hline & & 5.00 & 7.64 & 109.00 \\
\hline & \multirow{2}{*}{$\mathrm{Pb}$} & 0.00 & 8.33 & - \\
\hline & & 5.00 & 13.18 & 97.00 \\
\hline \multirow{4}{*}{ Yi Lake } & \multirow{2}{*}{$\mathrm{Cd}$} & 0.00 & 14.96 & \\
\hline & & 5.00 & 19.74 & 95.60 \\
\hline & \multirow{2}{*}{$\mathrm{Pb}$} & 0 & 35.30 & - \\
\hline & & 5.00 & 40.65 & 107.00 \\
\hline
\end{tabular}




\section{Conclusion}

The present study focused on the development of a new method to enrich trace cadmium and lead in environmental water samples using a novel PS-CSC adsorbent. The results demonstrate the feasibility of using PS-CSC adsorbent for preconcentration of cadmium and lead from aqueous solutions. The adsorption efficiency of cadmium and lead are $\mathrm{pH}$, time, and elution condition dependent. This enrichment method shows fast metal sorption kinetics and high adsorption efficiency. The maximum adsorption capacities of cadmium and lead on PS-CSC sorbent are 6.03-18.17 mg/g and $12.58-36.13 \mathrm{mg} / \mathrm{g}$ when the initial concentration of metal ions is between $5.0-90 \mathrm{mg} / \mathrm{L}$; the optimal $\mathrm{pH}$ values for quantitative sorption were $5.0-8.0$ and $7.0-10.0$ for cadmium and lead, respectively. These results indicate that the present method is accurate and precise. On the basis of the results obtained in this study, we believe that PS-CSC sorbent can be used for sorption of trace amounts of cadmium and lead from environmental water samples.

\section{Acknowledgment}

This work was jointly supported by the National Natural Science Foundation of China (21107001), the National Science and Technology Support Project of China (2011BAJ 03B04), The China Postdoctoral Science Foundation (2013 M541842), the Natural Science Fund of Education Department of Anhui Province (KJ2013Z034), and the Doctor Foundation of Anhui Jianzhu University (2012).

\section{REFERENCES}

1. B. Benguella and H. Benaissa, "Cadmium Removal from Aqueous Solutions by Chitin: Kinetic and Equilibrium Studies," Water Res., 36 2463-74 (2002).

2. R. Rivas, I. López-García, and M. Hernández-Córdoba, "Determination of Traces of Lead and Cadmium Using Dispersive Liquid-liquid Microextraction Followed by Electrothermal Atomic Absorption Spectrometry," Microchim. Acta., 166 355-61 (2009).

3. F. Xie, X. Lin, X. Wu, and Z. Xie, "Solid Phase Extraction of Lead (II), Copper (II), Cadmium (II) and Nickel (II) Using Gallic Acid-modified Silica Gel Prior to Determination by Flame Atomic Absorption Spectrometry," Talanta, 74 83643 (2008).

4. N. Burham, S. M. Abdel-Azeem, and F. El-Shahat, "Determination of Lead and Cadmium in Tap Water and Apple Leaves after Preconcentration on a New Acetylacetone Bonded Polyurethane foam Sorbent," Inter. J. Environ. Anal. Chem., 88 775-89 (2008).
5. L. A. Portugal, H. S. Ferreira, W. N. dos Santos, and S. L. Ferreira, "Simultaneous Pre-concentration Procedure for the Determination of Cadmium and Lead in Drinking Water Employing Sequential Multi-element Flame Atomic Absorption Spectrometry," Microchem. J., 87 77-80 (2007).

6. H. Parham, N. Pourreza, and N. Rahbar, "Solid Phase Extraction of Lead and Cadmium Using Solid Sulfur as a New Metal Extractor Prior to Determination by Flame Atomic Absorption Spectrometry," J. Hazard. Mater., 163 588-92 (2009).

7. G. Doner and A. Ege, "Determination of Copper, Cadmium and Lead in Seawater and Mineral Water by Flame Atomic Absorption Spectrometry after Coprecipitation with Aluminum Hydroxide," Anal. Chim. Acta., 547 14-17 (2005).

8. Ş. Tokalığlu, T. Oymak, and Ş. Kartal, "Coprecipitation of Lead and Cadmium Using Copper (II) Mercaptobenzothiazole Prior to Flame Atomic Absorption Spectrometric Determination," Microchim. Acta., 159 133-39 (2007).

9. F. A. Amorim and S. L. Ferreira, "Determination of Cadmium and Lead in Table Salt by Sequential Multi-element Flame Atomic Absorption Spectrometry," Talanta, 65 96064 (2005).

10. A. N. Anthemidis and K.-I. G. Ioannou, "On-line Sequential Injection Dispersive Liquid-liquid Microextraction System for Flame Atomic Absorption Spectrometric Determination of Copper and Lead in Water Samples," Talanta, 79 86-91 (2009).

11. J. Chen and K. C. Teo, "Determination of Cadmium, Copper, Lead and Zinc in Water Samples by Flame Atomic Absorption Spectrometry after Cloud Point Extraction," Anal. Chim. Acta., 450 215-22 (2001).

12. M. Ghaedi, A. Shokrollahi, K. Niknam, E. Niknam, A. Najibi, and M. Soylak, "Cloud Point Extraction and Flame Atomic Absorption Spectrometric Determination of Cadmium (II), Lead (II), Palladium (II) and Silver (I) in Environmental Samples," J. Hazard. Mater., 168 1022-27 (2009).

13. C. Duran, A. Gundogdu, V. N. Bulut, M. Soylak, L. Elci, H. B. Sentürk, and M. Tüfekci, "Solid-phase Extraction of Mn (II), Co (II), Ni (II), Cu (II), Cd (II) and Pb (II) Ions from Environmental Samples by Flame Atomic Absorption Spectrometry (FAAS),” J. Hazard. Mater., 146 347-55 (2007).

14. J. J. Sun and J. S. Fritz, "Chemically Modified Resins for Solid-phase Extraction,” J. Chromatogr. A, 590 197-202 (1992).

15. R. Ando, H. Inden, M. Sugino, H. Ono, D. Sakaeda, T. Yagyu, and M. Maeda, "Spectroscopic Characterization of Amino Acid and Amino Acid Ester-Schiff-base Complexes of Oxovanadium and Their Catalysis in Sulfide Oxidation," Inorg. Chim. Acta., 357 1337-44 (2004).

16. D. Prabhakaran and M. Subramanian, "A Column System for the Selective Extraction of U (VI) and Th (IV) Using a nEw Chelating Sorbent," Talanta, 61 423-30 (2003). 